Article

\title{
Bioactive Chemical Constituents from the Brown Alga Homoeostrichus formosana
}

\author{
Hui-Yu Fang ${ }^{1, \dagger}$, Uvarani Chokkalingam ${ }^{1, \dagger}$, Shu-Fen Chiou ${ }^{1}$, Tsong-Long Hwang ${ }^{2,3}$, \\ Shu-Li Chen ${ }^{4}$, Wei-Lung Wang ${ }^{5}$ and Jyh-Horng Sheu ${ }^{1,4,6, *}$
}

1 Department of Marine Biotechnology and Resources, National Sun Yat-sen University, Kaohsiung 80424, Taiwan; E-Mails: ahui0220@yahoo.com.tw (H.-Y.F.); uvaranichem@gmail.com (U.C.); sfc@mail.nsysu.edu.tw (S.-F.C.)

2 Graduate Institute of Natural Products, Chang Gung University, Taoyuan 33302, Taiwan; E-Mail: ht1@mail.cgu.edu.tw

3 Chinese Herbal Medicine Research Team, Healthy Aging Research Center, Chang Gung University, Taoyuan 33302, Taiwan

4 Graduate Institute of Natural Products, Kaohsiung Medical University, Kaohsiung 80708, Taiwan; E-Mail: slchen@kmu.edu.tw

5 Department of Biology, National Changhua University of Education, Changhua 50007, Taiwan; E-Mail: wlwang@cc.ncue.edu.tw

6 Department of Medical Research, China Medical University Hospital, China Medical University, Taichung 40402, Taiwan

$\dagger$ These authors contributed equally to this work.

* Author to whom correspondence should be addressed; E-Mail: sheu@mail.nsysu.edu.tw; Tel.: +886-7-525-2000 (ext. 5030); Fax: +886-7-525-5020.

Academic Editor: Chang Won Choi

Received: 18 November 2014 / Accepted: 17 December 2014 / Published: 30 December 2014

Abstract: A new chromene derivative, 2-(4',8'-dimethylnona-3'E,7'-dienyl)-8-hydroxy-2,6dimethyl-2H-chromene (1) together with four known natural products, methylfarnesylquinone (2), isololiolide (3), pheophytin a (4), and $\beta$-carotene (5) were isolated from the brown alga Homoeostrichus formosana. The structure of 1 was determined by extensive 1D and 2D spectroscopic analyses. Acetylation of $\mathbf{1}$ yielded the monoacetylated derivative 2-(4',8'-dimethylnona-3'E,7'-dienyl)-8-acetyl-2,6-dimethyl- $2 H$-chromene (6). Compounds 1-6 exhibited various levels of cytotoxic, antibacterial, and anti-inflammatory activities. Compound 2 was found to display potent in vitro anti-inflammatory activity by inhibiting 
the generation of superoxide anion ( $\mathrm{IC}_{50} 0.22 \pm 0.03 \mu \mathrm{g} / \mathrm{mL}$ ) and elastase release ( $\left.\mathrm{IC}_{50} 0.48 \pm 0.11 \mu \mathrm{g} / \mathrm{mL}\right)$ in FMLP/CB-induced human neutrophils.

Keywords: Homoeostrichus formosana; chromenes; cytotoxicity; antibacterial; anti-inflammatory

\section{Introduction}

In continuation of our search for bioactive chemical constituents from marine alga [1-6], we found that a crude acetone extract of Homoeostrichus formosana (Dictyotaceae, Phaeophyceae) [7] displayed significant cytotoxic and anti-inflammatory properties. A novel chromene 2-(4',8'-dimethylnona-3' $E, 7$ '-dienyl)-8-hydroxy-2,6-dimethyl-2 $H$-chromene (1), along with four known compounds methylfarnesylquinone (2) [8], isololiolide (3) [9], pheophytin a (4) [10,11], and $\beta$-carotene (5) [12] (Chart 1) were identified for the first time using a bioassay-directed fractionation method. Meroterpenoids of the chromene skeleton and related structural units incorporated with a polyprenyl chain, are particularly abundant in brown alga (division Phaeophyta), which makes chromenes one of the representative groups of secondary metabolites of these organisms [13-15]. 2-Substituted $2 \mathrm{H}$-chromenes and their analogs are pervasive molecular structures in chemistry, biology, and medicine. Many of these compounds possess various interesting biological properties that includes antidiabetic [16], cytotoxic [17], antifungal [18], anti-HIV [19], antibacterial [20], antitumor [21] and potent antioxidant (vitamin E) [22,23] activities. Recently, we also reported cytotoxic, anti-inflammatory, and antibacterial properties of sulfur-containing polybromoindoles from the Formosan red alga Laurencia brongniartii [24]. Consequently, the study has been undertaken to assess the cytotoxic, antibacterial, and anti-inflammatory activities of isolated compounds 1-6. Compounds 1, 2, and 4-6 were tested for cytotoxicity against HepG2, A-549, and MDA-MB-231 cancer cell lines as well as antibacterial activity against Bacillus cereus, Staphylococcus aureus, Salmonella typhimurium, Pseudomonas aeruginosa, Serratia marcescens and Yersinia entercocolitica. The abilities of $\mathbf{1}, \mathbf{2}, \mathbf{5}$, and $\mathbf{6}$ to inhibit superoxide anion generation and elastase release in FMLP/CB induced human neutrophils were also studied. Herein, we describe the isolation and structure elucidation of compound $\mathbf{1}$, and the results of biological activity assays of compounds 1-6.

\section{Results and Discussion}

Compound 1 was obtained as a brown oil and its molecular formula was assigned as $\mathrm{C}_{22} \mathrm{H}_{30} \mathrm{O}_{2}$ by HRESIMS (High Resolution Electron Spray Ionization Mass Spectrometry) showing a $[\mathrm{M}+\mathrm{Na}]^{+}$peak at $m / z$ 349.2143, implying eight degrees of unsaturation. The UV spectrum showed absorption maxima at 206, 231, 265, 274 and $333 \mathrm{~nm}$, suggesting a conjugated aromatic ring system [25] and IR absorption bands indicated the presence of hydroxy $\left(3396 \mathrm{~cm}^{-1}\right)$, and an aromatic chromophore (1593, $1463 \mathrm{~cm}^{-1}$ ) [26]. The ${ }^{13} \mathrm{C}$-NMR spectrum (Figure S1) comprises resonances of 22 carbon signals and these signals were assigned to five methyls, four $\mathrm{sp}^{3}$ methylenes, six $\mathrm{sp}^{2}$ methines, six $\mathrm{sp}^{2}$ quaternary carbons and one $\mathrm{sp}^{3}$ quaternary carbon using a combination of DEPT (Distortionless Enhancement by 
Polarization Transfer) and HSQC (Heteronuclear Single Quantum Coherence) data (Figures S2 and S3). The characteristic $2 H$-chromene moiety was inferred by the aromatic proton resonances $(\delta 6.34,1 \mathrm{H}, \mathrm{d}$, $J=2.8 \mathrm{~Hz} ; 6.49,1 \mathrm{H}, \mathrm{d}, J=2.8 \mathrm{~Hz})$, and AB type doublets $(\delta 5.60,1 \mathrm{H}, \mathrm{d}, J=9.6 \mathrm{~Hz} ; 6.25,1 \mathrm{H}, \mathrm{d}$, $J=9.6 \mathrm{~Hz}$ ) in the ${ }^{1} \mathrm{H}-\mathrm{NMR}$ spectrum (Figure S4), as well as eight $\mathrm{sp}^{2}$ carbons ( $\delta 110.3$ to 148.5) and one quaternary carbon at $\delta 77.8$ in the ${ }^{13} \mathrm{C}-\mathrm{NMR}$ spectrum. The ${ }^{1} \mathrm{H}-\mathrm{NMR}$ spectrum indicated the presence of a geranylmethyl moiety, as evidenced by the resonances at $\delta 1.72(2 \mathrm{H}, \mathrm{m}), 2.12$ $(2 \mathrm{H}, \mathrm{m}), 1.98(2 \mathrm{H}, \mathrm{m}), 2.06(2 \mathrm{H}, \mathrm{m}), 5.11$ to $5.14(2 \mathrm{H}$, overlapped) and three methyl singlets. Further, the constitution of this side chain was confirmed by ${ }^{1} \mathrm{H}-{ }^{1} \mathrm{H}$ COSY $\left({ }^{1} \mathrm{H}-{ }^{1} \mathrm{H}\right.$ correlation spectroscopy) (Figure S5) correlations from $\mathrm{H}_{2}-1^{\prime}$ to $\mathrm{H}_{2}-2^{\prime}, \mathrm{H}_{2}-2$ ' to $\mathrm{H}-3$ ', $\mathrm{H}_{2}-5$ ' to $\mathrm{H}_{2}-6$ ' and $\mathrm{H}_{2}-6$ ' to $\mathrm{H}_{-} 7^{\prime}$. The HMBC (Heteronuclear Multiple Bond Correlation) (Figure S6) spectrum exhibited interactions of the $\mathrm{H}_{3}-12$ $\left(\delta 1.38, \mathrm{CH}_{3}\right)$ with $\mathrm{C}-2(\delta 77.8), \mathrm{C}-3\left(\delta\right.$ 130.7) and C-1' $(\delta 40.7)$, and the $\mathrm{H}-1{ }^{\prime}(\delta 1.72)$ with $\mathrm{C}-2$ $(\delta 77.8)$ and $\mathrm{C}-2^{\prime}(\delta 22.5)$. These HMBC data suggested attachment of the $\mathrm{C}-1$ ' of geranylmethyl at the $\mathrm{C}-2$ of $2 \mathrm{H}$-pyran ring. The methyl group substitution at $\mathrm{C}-6$ was inferred by HMBC interactions of $\mathrm{H}-7$ $\left(\delta\right.$ 6.49) to $\mathrm{C}-11\left(\delta 15.4, \mathrm{CH}_{3}\right)$. The complete ${ }^{1} \mathrm{H}$ - and ${ }^{13} \mathrm{C}-\mathrm{NMR}$ chemical shift assignments, ${ }^{1} \mathrm{H}-{ }^{1} \mathrm{H}$ COSY and HMBC correlations, are presented in Table 1 . The ${ }^{1} \mathrm{H}-$ and ${ }^{13} \mathrm{C}-\mathrm{NMR}$ spectroscopic data of $\mathbf{1}$ were similar to those of 2-(4',8'-dimethylnona-3',7'-dienyl)-8-hydroxy-2-methyl-2H-chromene-6-carboxylic acid methyl ester [27], which was previously isolated from Piper umbellatum and Piper peltatum. The only exception is that the methyl ester at the C-6 position in 2-(4',8'-dimethylnona-3',7'-dienyl)-8hydroxy-2-methyl-2H-chromene-6-carboxylic acid methyl ester was replaced by a methyl group. Additionally, acetylation of $\mathbf{1}$ yielded the monoacetylated derivative $\mathbf{6}$, which displayed a $[\mathrm{M}+\mathrm{Na}]^{+}$ at 391.2249 in HRESIMS, thus a molecular formula of $\mathrm{C}_{24} \mathrm{H}_{32} \mathrm{O}_{3}$ was proposed for 6. Indeed, the resonance due to $\mathrm{H}-7$ was observed at $\delta 6.68$ (C-7/ $\delta$ 122.8), showing the expected downfield shift in comparison to $\mathbf{1}$ and thus confirmed the location of acetyl group at C-8. The geometry of double bond $\mathrm{C}-3^{\prime} / \mathrm{C}-4^{\prime}$ of geranylmethyl was assigned as $E$ on the basis of the NOESY correlations of $\mathrm{H}_{2}-2^{\prime} / \mathrm{H}_{3}-11^{\prime}$ and $\mathrm{H}-3^{\prime} / \mathrm{H}_{2}-5^{\prime}$. Moreover, compound $\mathbf{1}$ did not show any significant Cotton effects and its poor optical activity indicates possibly it is a 1:1 mixture of two enantiomers [25]. Based on the above evidences and detailed analyses of NMR spectra, the structure of compound $\mathbf{1}$ was determined as 2-(4',8'-dimethylnona-3' $E$,7'-dienyl)-8-hydroxy-2,6-dimethyl-2H-chromene.

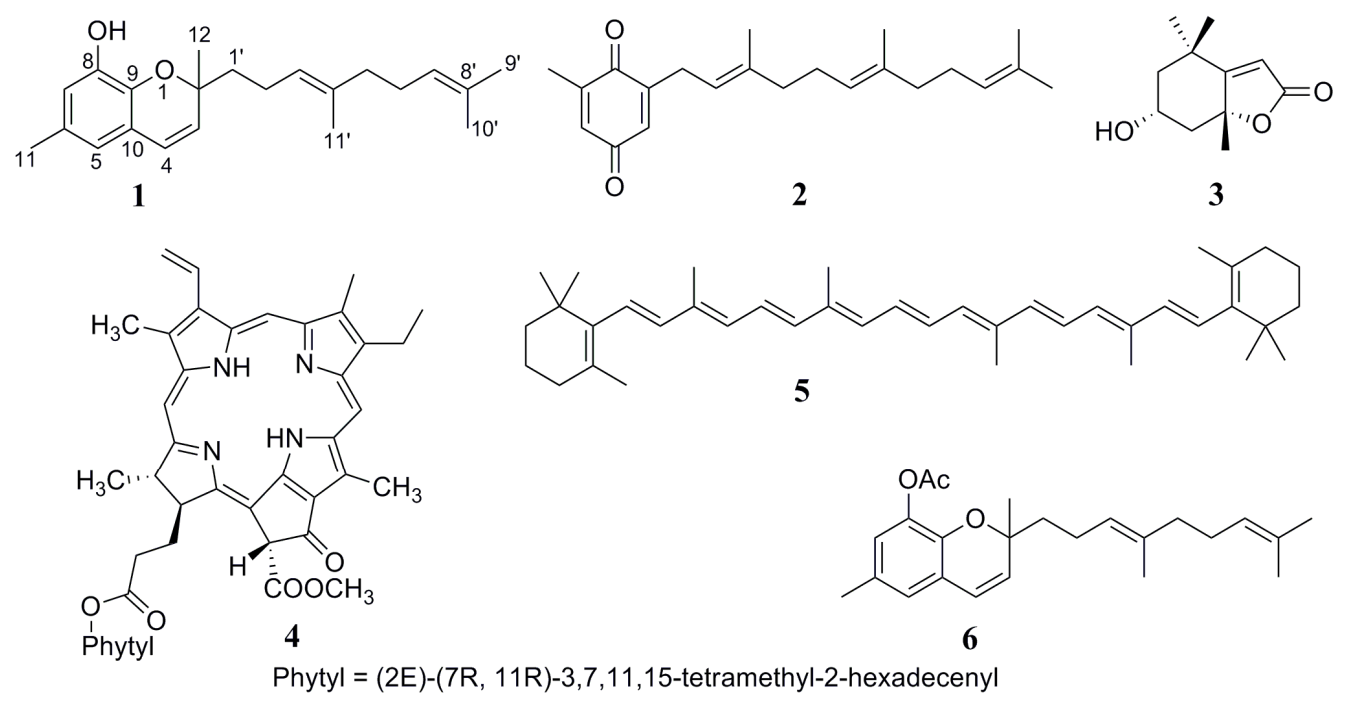

Chart 1. Structures of metabolites 1-6. 
This chemical investigation also afforded four known compounds, methylfarnesylquinone (2) [8], isololiolide (3) [9], pheophytin a (4) [10,11], and $\beta$-carotene (5) [12]. Compounds 2-5 were identified by comparing their UV, IR and NMR spectroscopic data with those reported in the literature.

Table 1. ${ }^{1} \mathrm{H}$ - and ${ }^{13} \mathrm{C}-\mathrm{NMR}$ data, ${ }^{1} \mathrm{H}-{ }^{1} \mathrm{H}$ COSY, and HMBC correlations of $\mathbf{1}$.

\begin{tabular}{|c|c|c|c|c|}
\hline Position & ${ }^{1} \mathbf{H}^{a}$ & ${ }^{13} \mathrm{C}^{b}$ & ${ }^{1} \mathrm{H}-{ }^{1} \mathrm{H} \mathrm{COSY}$ & НMBC \\
\hline 2 & & $77.8(\mathrm{C})$ & & \\
\hline 3 & $5.60 \mathrm{~d}(9.6)$ & $130.7(\mathrm{CH})^{d}$ & $\mathrm{H}-4$ & $C-2, C-10$ \\
\hline 4 & $6.25 \mathrm{~d}(9.6)^{c}$ & $122.8(\mathrm{CH})$ & $\mathrm{H}-3$ & $\mathrm{C}-2, \mathrm{C}-5, \mathrm{C}-8, \mathrm{C}-10$ \\
\hline 5 & $6.34 \mathrm{~d}(2.8)$ & $110.3(\mathrm{CH})$ & & C-4, C-7, C-8 \\
\hline 6 & & $126.3(\mathrm{C})$ & & \\
\hline 7 & $6.49 \mathrm{~d}(2.8)$ & $117.1(\mathrm{CH})$ & & C-5, C-8, C-11 \\
\hline 8 & & $144.8(\mathrm{C})$ & & \\
\hline 9 & & $148.5(\mathrm{C})$ & & \\
\hline 10 & & $121.4(\mathrm{C})$ & & \\
\hline 11 & $2.15 \mathrm{~s}$ & $15.4\left(\mathrm{CH}_{3}\right)$ & & $\mathrm{C}-6, \mathrm{C}-7, \mathrm{C}-8$ \\
\hline 12 & $1.38 \mathrm{~s}$ & $25.8\left(\mathrm{CH}_{3}\right)$ & & $\mathrm{C}-2, \mathrm{C}-3, \mathrm{C}-1^{\prime}$ \\
\hline $1^{\prime}$ & $1.72 \mathrm{~m}$ & $40.7\left(\mathrm{CH}_{2}\right)$ & $\mathrm{H}_{2}-2^{\prime}$ & $\mathrm{C}-2, \mathrm{C}-2^{\prime}$ \\
\hline $2^{\prime}$ & $2.12 \mathrm{~m}$ & $22.5\left(\mathrm{CH}_{2}\right)$ & $\mathrm{H}_{2}-1^{\prime}, \mathrm{H}-3^{\prime}$ & \\
\hline $3^{\prime}$ & 5.14 (overlapped with H-7') & $124.0(\mathrm{CH})$ & $\mathrm{H}_{2}-2^{\prime}$ & C-2', C-5', C-11' \\
\hline $4^{\prime}$ & & $135.2(\mathrm{C})$ & & \\
\hline $5^{\prime}$ & $1.98 \mathrm{~m}$ & $39.6\left(\mathrm{CH}_{2}\right)$ & $\mathrm{H}_{2}-6^{\prime}$ & C-4', C-6', C-7' \\
\hline $6^{\prime}$ & $2.06 \mathrm{~m}$ & $26.6\left(\mathrm{CH}_{2}\right)$ & $\mathrm{H}_{2}-5^{\prime}, \mathrm{H}-7^{\prime}$ & C-4', C-5', C-7' \\
\hline $7^{\prime}$ & 5.11 (overlapped with H-3') & $124.3(\mathrm{CH})$ & $\mathrm{H}_{2}-6^{\prime}$ & C-6', C-9', C-10' \\
\hline $8^{\prime}$ & & $131.3(\mathrm{C})$ & & \\
\hline $9^{\prime}$ & $1.70 \mathrm{~s}$ & $25.6\left(\mathrm{CH}_{3}\right)$ & & C-7', C-10' \\
\hline $10^{\prime}$ & $1.61 \mathrm{~s}$ & $17.6\left(\mathrm{CH}_{3}\right)$ & & C-7', C-8' \\
\hline $11^{\prime}$ & $1.60 \mathrm{~s}$ & $15.8\left(\mathrm{CH}_{3}\right)$ & & C-4', C-5' \\
\hline $8-\mathrm{OH}$ & $5.35 \mathrm{~s}$ & & & \\
\hline
\end{tabular}

${ }^{a}$ Spectra recorded at $400 \mathrm{MHz}$ in $\mathrm{CDCl}_{3}$ at $25{ }^{\circ} \mathrm{C} ;{ }^{b}$ Spectra recorded at $100 \mathrm{MHz}$ in $\mathrm{CDCl}_{3}$ at $25{ }^{\circ} \mathrm{C}$;

${ }^{c} J$ values (in Hz)in parentheses; ${ }^{d}$ Attached protons were deduced by DEPT experiments.

Compounds 1, 2 and 4-6 were tested for cytotoxic effects against HepG2, A-549, and MDA-MB-231 cancer cell lines using the MTT assay. As shown in Table 2, compounds 1, 2 and $\mathbf{6}$ revealed moderate to weak cytotoxicity with $\mathrm{IC}_{50}$ values ranging from 20.2 to $43.1 \mu \mathrm{M}$ and the remaining compounds were inactive. These results revealed that hydroxy chromene (1) was more effective than its acetyl derivative (6). Compound 1 exhibited significant antibacterial activity (Table 3) against $S$. marcescens with a dosage of $10 \mu \mathrm{g} /$ disk (Inhibition zone: $1.0 \mathrm{~mm}$ ) when compared to that of standard antibiotic ampicillin with a dosage of $100 \mu \mathrm{g}$ /disk (Inhibition zone: $1.5 \mathrm{~mm}$ ). In addition, compounds $\mathbf{1}, \mathbf{2}, \mathbf{5}$ and 6 showed good potential against $P$. aeruginosa compared to that of ampicillin. 
Table 2. Cytotoxicity data of compounds 1, 2 and 4-6.

\begin{tabular}{cccc}
\hline \multirow{2}{*}{ Compounds } & \multicolumn{3}{c}{ Cell Lines, $\mathbf{I C}_{\mathbf{5 0}}(\boldsymbol{\mu M})$} \\
\cline { 2 - 4 } & Hep G2 & A549 & MDA-MB-231 \\
\hline $\mathbf{1}$ & 22.4 & 43.1 & 20.2 \\
$\mathbf{2}$ & 30.1 & 27.0 & 26.0 \\
$\mathbf{4}$ & $-\mathrm{a}$ & - & - \\
$\mathbf{5}$ & - & - & - \\
$\mathbf{6}$ & 41.5 & 38.2 & 35.9 \\
Doxorubicin ${ }^{\mathrm{b}}$ & 0.3 & 1.9 & 2.1 \\
\hline${ }^{\mathrm{a}}$ Inactive with $\mathrm{IC}_{50}>50 \mu \mathrm{M}^{\mathrm{b}}{ }^{\mathrm{b}}$ Doxorubicin was used as a reference compound.
\end{tabular}

Table 3. Antibacterial activity (zone of inhibition in $\mathrm{mm}$ ) of compounds 1, 2 and 4-6.

\begin{tabular}{|c|c|c|c|c|c|c|c|}
\hline \multirow[b]{2}{*}{ Compounds } & \multirow[b]{2}{*}{$\begin{array}{c}\text { Dosage } \\
\text { ( } \mu \mathrm{g} / \text { disk })\end{array}$} & \multicolumn{6}{|c|}{ Inhibition Zone (mm) } \\
\hline & & $\begin{array}{c}\text { B. } \\
\text { cereus }\end{array}$ & $\begin{array}{c}S . \\
\text { aureus }\end{array}$ & $\begin{array}{c}S . \\
\text { typhimurium }\end{array}$ & $\begin{array}{c}P . \\
\text { aeruginosa }\end{array}$ & $\begin{array}{c}S . \\
\text { marcescens }\end{array}$ & $\begin{array}{c}Y . \\
\text { enterocolitica }\end{array}$ \\
\hline \multirow[t]{7}{*}{1} & 100 & & & & 2.5 & & \\
\hline & 40 & 1.0 & 3.0 & 0.5 & & 1.5 & 2.0 \\
\hline & 30 & 0.5 & 1.0 & 0.5 & & 1.5 & 1.5 \\
\hline & 20 & 2.0 & 3.5 & 1.5 & & 3.0 & 1.0 \\
\hline & 15 & 1.5 & 2.5 & 1.5 & & 2.5 & 2.0 \\
\hline & 10 & 1.0 & 2.0 & 2.0 & & 1.0 & 2.0 \\
\hline & 5 & & & & & & 1.0 \\
\hline \multirow[t]{3}{*}{2} & 60 & & & & 1.0 & & \\
\hline & 50 & & & & 0.5 & & \\
\hline & 30 & & & & 0.5 & & \\
\hline \multirow[t]{4}{*}{4} & 20 & & & & & & 2.5 \\
\hline & 15 & & & & & & 1.0 \\
\hline & 10 & & & & & & 0.5 \\
\hline & 5 & & & & & & 0.5 \\
\hline \multirow[t]{2}{*}{5} & 30 & & & & 0.5 & & \\
\hline & 20 & & & & 0.5 & & \\
\hline \multirow[t]{4}{*}{6} & 60 & & & 1.0 & & & \\
\hline & 50 & & & 1.0 & & & \\
\hline & 30 & & & 0.5 & & & \\
\hline & 20 & & & & 1.0 & & \\
\hline \multirow[t]{5}{*}{ Ampicillin } & 300 & 4.0 & 13.0 & 10.0 & 0.0 & 4.0 & \\
\hline & 200 & 3.5 & 13.0 & 8.5 & 0.0 & 2.5 & \\
\hline & 100 & 3.5 & 12.0 & 6.5 & 0.0 & 1.5 & 18.0 \\
\hline & 50 & 3.0 & 10.5 & 5.0 & 0.0 & & 11.0 \\
\hline & 25 & & 8.5 & 4.0 & 0.0 & & 4.0 \\
\hline
\end{tabular}

The in vitro anti-inflammatory activity of compounds $1,2,5$, and $\mathbf{6}$ were evaluated by suppressing $N$-formyl-methionyl-leucylphenylalanine/cytochalasin B (FMLP-CB)-induced superoxide anion generation and elastase release in human neutrophils (Table 4). Among them, compound $\mathbf{2}$ was found to display 
potent inhibitory effects on generation of superoxide anion ( $\mathrm{IC}_{50} 0.22 \pm 0.03 \mu \mathrm{g} / \mathrm{mL}$ ) and the release of elastase ( $\mathrm{IC}_{50} 0.48 \pm 0.11 \mu \mathrm{g} / \mathrm{mL}$ ) by human neutrophils at a concentration of $10 \mu \mathrm{g} / \mathrm{mL}$.

Table 4. Inhibitory effects of compounds $1,2,5$ and 6 on superoxide anion generation and elastase release in FMLP/CB-induced human neutrophils at $10 \mu \mathrm{g} / \mathrm{mL}$.

\begin{tabular}{ccccc}
\hline \multirow{2}{*}{ Compounds } & \multicolumn{2}{c}{ Superoxide Anion } & \multicolumn{2}{c}{ Elastase Release } \\
\cline { 2 - 5 } & $\mathbf{I C}_{\mathbf{5 0}}(\boldsymbol{\mu \mathbf { g }} / \mathbf{m L})^{\mathbf{a}}$ & $\mathbf{I n h} \mathbf{\%}$ & $\mathbf{I C}_{\mathbf{5 0}}(\boldsymbol{\mu} \mathbf{g} / \mathbf{m L})^{\mathbf{a}}$ & $\mathbf{I n h} \%$ \\
\hline $\mathbf{1}$ & & $-16.34 \pm 1.87 * * *$ & & $-30.28 \pm 1.49 * * *$ \\
$\mathbf{2}$ & $0.22 \pm 0.03$ & $91.63 \pm 1.30 * * *$ & $0.48 \pm 0.11$ & $117.22 \pm 6.53 * * *$ \\
$\mathbf{5}$ & $>10$ & $38.42 \pm 5.79 * *$ & $>10$ & $43.48 \pm 4.36^{* * *}$ \\
$\mathbf{6}$ & $>10$ & $8.31 \pm 3.25$ & $>10$ & $14.62 \pm 3.84 *$ \\
\hline
\end{tabular}

Percentage of inhibition (Inh \%) at $10 \mu \mathrm{M}$ concentration. Results are presented as mean \pm S.E.M. (the standard error of mean) ( $n=3$ or 4$)$. ${ }^{*} p<0.05,{ }^{* *} p<0.01,{ }^{* * *} p<0.001$ compared with the control value.

${ }^{\text {a }}$ Concentration necessary for $50 \%$ inhibition $\left(\mathrm{IC}_{50}\right)$.

\section{Experimental Section}

\subsection{General Experimental Procedures}

Optical rotations were measured on a JASCO P1020 polarimeter (JASCO, Tokyo, Japan). IR spectra were recorded on a JASCO FT/IR-4100 spectrophotometer (JASCO, Tokyo, Japan). Ultraviolet spectra were recorded on a JASCO V-650 spectrophotometer (JASCO, Tokyo, Japan). The NMR spectra were recorded in $\mathrm{CDCl}_{3}$ on a Varian MR-400 FT-NMR spectrometer (Varian, Palo Alto, CA, USA). ESIMS and HRESIMS were recorded by ESI FT-MS on a Bruker APEX II mass spectrometer (Bruker, Bremen, Germany). Silica gel 60 (230-400 mesh, Merck, Darmstadt, Germany) was used for column chromatography. Pre-coated silica gel plates (Kieselgel $60 \mathrm{~F}_{254} 0.25 \mathrm{~mm}$, Merck) were used for TLC analyses. High-performance liquid chromatography was performed on a Hitachi L-7100 HPLC apparatus (Hitachi, Tokyo, Japan) with a C-18 column $(250 \mathrm{~mm} \times 10 \mathrm{~mm}, 5 \mu \mathrm{m}$, Supelco, Bellefonte, PA, USA).

\subsection{Alga Material}

The brown alga $H$. formosana was collected by hand via scuba off the coast of San-Hsian-Tai, in July 2008, at a depth of 2-5 m, and stored in a freezer until extraction. A voucher specimen (2008-SHTBA-001) was deposited in the Department of Marine Biotechnology and Resources, National Sun Yat-sen University.

\subsection{Extraction and Isolation}

The frozen bodies of $H$. formosana ( $1.3 \mathrm{~kg}$, wet weight) were minced and exhaustively extracted with acetone $(10 \times 1.5 \mathrm{~L}$, each for 3 days $)$ at room temperature. The acetone extract $(10.3 \mathrm{~g})$ was fractionated by open column chromatography on silica gel using $n$-hexane-EtOAc and EtOAc-MeOH mixtures of increasing polarity to yield 40 fractions. Fraction 4 was eluted with $n$-hexane-EtOAc (80:1) to afford $5(12.1 \mathrm{mg})$. Fraction 9, eluted with $n$-hexane-EtOAc (30:1), was subjected to a Sephadex LH-20 column, using acetone as the mobile phase, to afford 2 (33.5 mg). Fraction 13, 
eluting with $n$-hexane-EtOAc (4:1), was subjected to a Sephadex LH-20 column, using acetone as mobile phase, to afford five separated subfractions. Subfraction 3 was further separated by silica gel column ( $n$-hexane-Acetone, 10:1) to afford $1(65.8 \mathrm{mg})$. Fraction 15, eluted with $n$-hexane-EtOAc (1:1), was subjected to a Sephadex LH-20 column, using acetone as mobile phase, to afford three separated subfractions. Subfraction 1 was further purified by silica gel column ( $n$-hexane-dichloromethane-acetone, 10:1:1) to afford $4(3.0 \mathrm{mg})$. Fraction 18 , eluted with $n$-hexane-EtOAc (1:2), was subjected to a Sephadex LH-20 column, using acetone as mobile phase, to afford five separated subfractions. Subfraction 4 was further separated by silica gel column ( $n$-hexane-dichloromethane-acetone, 10:1:1) to afford 3 (1.5 mg).

2-(4',8'-Dimethylnona-3'E,7'-dienyl)-8-hydroxy-2,6-dimethyl-2H-chromene (1): Pale yellow oil; $[\alpha]_{\mathrm{D}}^{25}=+0.5\left(c 0.25, \mathrm{CHCl}_{3}\right) ; \mathrm{UV}(\mathrm{MeOH}) \lambda_{\max }(\log \varepsilon) 206$ (4.4), 231 (4.3), 265 (3.6), 274 (3.6), $333(3.4) \mathrm{nm}$; IR (KBr) $v_{\max } 3396,2922,1729,1593,1463,1311,853$, and $717 \mathrm{~cm}^{-1} ;{ }^{13} \mathrm{C}-$ and

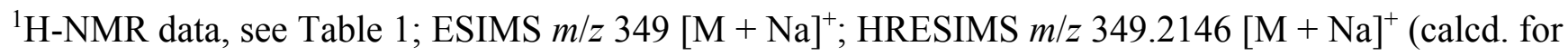
$\mathrm{C}_{22} \mathrm{H}_{30} \mathrm{O}_{2} \mathrm{Na}, 349.2143$ ).

\subsection{Acetylation of Compound 1}

Compound 1 (5.0 mg) was dissolved in pyridine $(0.2 \mathrm{~mL})$ and then $\mathrm{Ac}_{2} \mathrm{O}(0.1 \mathrm{~mL})$ was added. After $24 \mathrm{~h}$ at room temperature, the reaction residue was subjected to column chromatography over silica gel using $n$-hexane-EtOAc $(5: 1)$ to yield the acetyl derivative $6(4.1 \mathrm{mg})$.

2-(4',8'-Dimethylnona-3' $E, 7^{\prime}$-dienyl)-8-acetyl-2,6-dimethyl-2H-chromene (6): Colorless oil; $[\alpha]_{\mathrm{D}}^{25}=+0.3$ (c 0.1, $\mathrm{CHCl}_{3}$ ); UV (MeOH) $\lambda_{\max }(\log \varepsilon) 205$ (4.5), 227 (4.4), 266 (3.6), 275 (3.5), 318 (3.4) nm; IR (neat) $v_{\max } 2922,1760,1468,1369,1207,1020,894$, and $723 \mathrm{~cm}^{-1} ;{ }^{1} \mathrm{H}-\mathrm{NMR}\left(400 \mathrm{MHz}, \mathrm{CDCl}_{3}\right)$ : $\delta 5.58(1 \mathrm{H}, \mathrm{d}, J=9.6 \mathrm{~Hz}, \mathrm{H}-3), 6.27(1 \mathrm{H}, \mathrm{d}, J=9.6 \mathrm{~Hz}, \mathrm{H}-4), 6.68(1 \mathrm{H}, \mathrm{d}, J=2.8 \mathrm{~Hz}, \mathrm{H}-7), 6.55(1 \mathrm{H}$, $\mathrm{d}, J=2.8 \mathrm{~Hz}, \mathrm{H}-5), 2.16\left(3 \mathrm{H}, \mathrm{s}, 6-\mathrm{CH}_{3}\right), 1.38\left(3 \mathrm{H}, \mathrm{s}, 2-\mathrm{CH}_{3}\right), 2.16(3 \mathrm{H}, \mathrm{s}, 8-\mathrm{OAc}), 1.71$ (2H, m, H-1'), $2.11\left(2 \mathrm{H}, \mathrm{m}, \mathrm{H}-2^{\prime}\right), 5.14$ (1H, overlapped, H-3'), 1.97 (2H, m, H-5'), 2.05 (2H, m, H-6'), $5.11(1 \mathrm{H}$, overlapped, $\mathrm{H}-7$ '); ${ }^{13} \mathrm{C}-\mathrm{NMR}\left(100 \mathrm{MHz}, \mathrm{CDCl}_{3}\right): \delta 78.5$ (C-2), 130.3 (C-3), 122.5 (C-4), 116.5 (C-5), 126.3 (C-6), 122.8 (C-7), 148.7 (C-8), 143.3 (C-9), 120.9 (C-10), 26.5 (C-3/CH3), $15.5\left(\mathrm{C}-6 / \mathrm{CH}_{3}\right)$, 21.1 (C-8/OAc), 41.2 (C-1'), 22.6 (C-2'), 123.9 (C-3'), 135.3 (C-4'), 39.7 (C-5'), 26.7 (C-6'), 124.3 (C-7'),

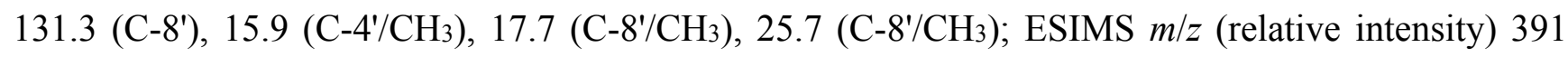
$[\mathrm{M}+\mathrm{Na}]^{+}$; HRESIMS m/z $391.2249[\mathrm{M}+\mathrm{Na}]^{+}$(calcd. for $\mathrm{C}_{24} \mathrm{H}_{32} \mathrm{O}_{3} \mathrm{Na}, 391.2246$ ).

\subsection{Cytotoxicity Testing}

Cell lines were purchased from the American Type Culture Collection (ATCC). Cytotoxicity assays of compounds 1, 2 and 4-6 were performed using the MTT [3-(4,5-dimethylthiazol-2-yl)-2,5diphenyl-tetrazolium bromide] colorimetric method [28,29].

\subsection{In Vitro Antibacterial Assay}

The antibacterial activity of the isolated compounds was evaluated against Bacillus cereus (ATCC14579), Staphylococcus aureus (ATCC9144), Salmonella typhimurium (ATCC914028), Pseudomonas aeruginosa (ATCC27853), Serratia marcescens (ATCC25419), and Yersinia enterocolitica 
(ATCC23715), based on previous reports [30,31]. Bacterial strains were grown in LB (Luria-Bertani) broth medium for $24 \mathrm{~h}$ at $37{ }^{\circ} \mathrm{C}$. Then, $17 \mathrm{~mL}$ LB hard agar (1.5\% agar) was poured into sterile Petri dishes $(90 \mathrm{~mm})$ and allowed to set. When testing the bacterial sample, $1000 \mu \mathrm{L}$ of inoculum suspension was poured into the molten LB soft agar plates using a sterile micropipet. After the temperature reached around $55-60{ }^{\circ} \mathrm{C}$, and the mixture was homogenized thoroughly by mixing in a circular motion (pour-plate technique). Sterile paper disks (Advantec, $8 \mathrm{~mm}$ in diameter) were placed onto the top layer of the LB agar plates. Test compounds $(2 \mu \mathrm{g} / \mu \mathrm{L})$ of different volumes were applied on each of the filter paper disks. Ampicillin $(5 \mu \mathrm{g} / \mu \mathrm{L})$ with different volumes of various dosages and DMSO were served as positive and negative controls respectively. All the plates were incubated at $37^{\circ} \mathrm{C}, 24 \mathrm{~h}$ prior to the evaluation of antibacterial activity.

\subsection{In Vitro Anti-Inflammatory Assay-Superoxide Anion Generation and Elastase Release by} Human Neutrophils

Human neutrophils were obtained by means of dextran sedimentation and Ficoll centrifugation. Measurements of superoxide anion generation and elastase release were carried out according to previously described procedures [32,33]. Briefly, superoxide anion production was assayed by monitoring the superoxide dismutase-inhibitable reduction of ferricytochrome c. Elastase release experiments were performed using MeO-Suc-Ala-Ala-Pro-Val-p-nitroanilide as the elastase substrate [34].

\section{Conclusions}

A new chromene derivative 2-(4',8'-dimethylnona-3'E,7'-dienyl)-8-hydroxy-2,6-dimethyl-2H-chromene (1) together with four known natural products 2-5 were isolated from the brown alga H. formosana. Both compounds 1 and 2 displayed marginal cytotoxicities toward HepG2, A-549, and MDA-MB-231 cancer cell lines. Compounds 1, 2, 5 and $\mathbf{6}$ showed potent inhibition against $P$. aeruginosa compared to that of ampicillin. Methylfarnesylquinone $\mathbf{2}$ revealed potent in vitro anti-inflammatory activity by inhibiting the generation of superoxide anion ( $\mathrm{IC}_{50} 0.22 \pm 0.03 \mu \mathrm{g} / \mathrm{mL}$ ) and the release of elastase ( $\mathrm{IC}_{50} 0.48 \pm 0.11 \mu \mathrm{g} / \mathrm{mL}$ ) in FMLP/CB-induced human neutrophils. Thus, compound 2 can be considered as a candidate for future anti-inflammatory drug development.

\section{Supplementary Materials}

Supplementary figures can be found at http://www.mdpi.com/1422-0067/16/01/0736/s1.

\section{Acknowledgments}

This research was supported by grants from the National Science Council of Taiwan (NSC 102-2320-B-110-001-MY2) and National Sun Yat-sen University-Kaohsiung Medical University Joint Project (NSYSU-KMU102-I-004), awarded to Jyh-Horng Sheu. 


\section{Author Contributions}

Jyh-Horng Sheu designed the research proposal and contributed to manuscript preparation. Hui-Yu Fang and Uvarani Chokkalingam carried out the experiments and wrote the manuscript. Shu-Fen Chiou, Tsong-Long Hwang and Shu-Li Chen contributed to carry out bioassays. Algal material was identified by Wei-Lung Wang.

\section{Conflicts of Interest}

The authors declare no conflict of interest.

\section{References}

1. Sheu, J.H.; Liaw, C.C.; Duh, C.Y. Oxygenated clerosterols isolated from the marine alga, Codium arabicum. J. Nat. Prod. 1995, 58, 1521-1526.

2. Sheu, J.H.; Huang, S.Y.; Duh, C.Y. Cytotoxic oxygenated desmosterols of the red alga, Galaxaura marginata. J. Nat. Prod. 1996, 59, 23-26.

3. Sheu, J.H.; Huang, S.Y.; Wang, G.H.; Duh, C.Y. Study on cytotoxic oxygenated desmosterols isolated from the red alga, Galaxaura marginata. J. Nat. Prod. 1997, 60, 900-903.

4. Sheu, J.H.; Wang, G.; Sung, J.; Duh, C.Y. New cytotoxic oxygenated fucosterols from the brown alga Turbinaria conoides. J. Nat. Prod. 1999, 62, 224-227.

5. Sheu, J.H.; Wang, G.H.; Sung, P.J.; Chiu, Y.H.; Duh, C.Y. Cytotoxic sterols from the formosan brown alga Turbinaria ornata. Planta Med. 1997, 63, 571-572.

6. Sheu, J.H.; Sung, P.J. Isolation of 24-hydroperoxy-24-vinylcholesterol and fucosterol from the brown alga Turbinaria conoides. J. Chin. Chem. Soc. 1991, 38, 501-503.

7. Wang, W.L.; Lin, C.S.; Lee, W.J.; Liu, S.L. Morphological and molecular characteristics of Homoeostrichus formosana sp. nov. (Dictyotaceae, Phaeophyceae) from Taiwan. Bot. Stud. 2013, 54, 1-13.

8. Naito, T.; Niitsu, K.; Ikeya, Y.; Okada, M.; Mitsuhashi, H. A phthalide and 2-farnesyl-6-methyl benzoquinone from Ligusticum chuangxiong. Phytochemistry 1992, 31, 1787-1789.

9. Kimura, J.; Maki, N. New loliolide derivatives from the brown alga Undaria pinnatifida. J. Nat. Prod. 2002, 65, 57-58.

10. Closs, G.L.; Katz, J.J.; Pennington, F.C.; Thomas, M.R.; Strain, H.H. Nuclear magnetic resonance spectra and molecular association of chlorophylls a and b, methyl chlorophyllides, pheophytins, and methyl pheophorbies. J. Am. Chem. Soc. 1963, 85, 3809-3821.

11. Nakatani, Y.; Ourisson, G.; Beck, J.J. Cytostatic pheophytins from silkworm excreta, and derive photocytotoxic pheophorbides. Chem. Pharm. Bull. 1981, 29, 2261-2269.

12. Lutnaes, B.F.; Bruas, L.; Kildahl-Andersen, G.; Krane, J.; Liaaen-Jensen, S. The charge delocalize $\beta, \beta$-carotene dication-preparation, structure eludication by NMR an reactions with nucleophiles. Org. Biomol. Chem. 2003, 1, 4064-4072.

13. Blunt, J.W.; Copp, B.R.; Munro, M.H.G.; Northcote, P.T.; Prinsep, M.R. Marine natural products. Nat. Prod. Rep. 2010, 27, 165-237. 
14. Blunt, J.W.; Copp, B.R.; Hu, W.P.; Munro, M.H.G.; Northcote, P.T.; Prinsep, M.R. Marine natural products. Nat. Prod. Rep. 2007, 24, 31-86.

15. Faulkner, D.J. Marine natural products. Nat. Prod. Rep. 1994, 11, 355-394.

16. Escandón-Rivera, S.; González-Andrade, M.; Bye, R.; Linares, E.; Navarrete, A.; Mata, R. $\alpha$-glucosidase inhibitors from Brickellia cavanillesii. J. Nat. Prod. 2012, 75, 968-974.

17. Chen, J.J.; Duh, C.Y.; Chen, I.S. Cytotoxic chromenes from Myriactis humilis. Planta Med. 2005, 71, 370-372.

18. Lago, J.H.G.; Ramos, C.S.; Casanova, D.C.C.; Morandim, A.A.; Bergamo, D.C.B.; Cavalheiro, A.J.; Bolzani, V.S.; Furlan, M.; Guimaraes, E.F.; Young, M.C.M.; et al. Benzoic acid derivatives from Piper species and their fungitoxic activity against Cladosporium cladosporioides and $C$. sphaerospermum. J. Nat. Prod. 2004, 67, 1783-1788.

19. Kurdyumov, A.V.; Hsung, R.P.; Ihlen, K.; Wang, J. Formal [3+3] cycloaddition approach to chromenes and chromanes. Concise total syntheses of $( \pm)$-rhododaurichromanic acids A and B and methyl ( \pm )-daurichromenic ester. Org. Lett. 2003, 5, 3935-3938.

20. Schneider, P.; Hawser, S.; Islam, K. Iclaprim, a novel diaminopyrimidine with potent activity on trimethoprim sensitive and resistant bacteria. Biorg. Med. Chem. Lett. 2003, 13, 4217-4221.

21. Gauthier, S.; Caron, B.; Cloutier, J.; Dory, Y.L.; Favre, A.; Larouche, D.; Mailhot, J.; Ouellet, C.; Schwerdtfeger, A.; Leblanc, G.; et al. (S)-(+)-4-[7-(2,2-Dimethyl-1-oxopropoxy)-4-methyl-2-[4[2-(1-piperidinyl)-ethoxy]phenyl]-2H-1-benzopyran-3-yl]-phenyl 2,2-dimethylpropanoate (EM-800): A highly potent, specific, and orally active nonsteroidal anti-estrogen. J. Med. Chem. 1997, 40, 2117-2122.

22. Seo, Y.; Park, K.E.; Kim, Y.A.; Lee, H.J.; Yoo, J.S.; Ahn, J.W.; Lee, B.J. Isolation of a new chromene from the brown alga Sargassum thunbergii. Bull. Korean Chem. Soc. 2007, 28, 1831-1833.

23. Mukai, K.; Okabe, K.; Hosose, H. Synthesis and stopped-flow investigation of antioxidant activity of tocopherols. Finding of new tocopherol derivatives having the highest antioxidant activity among phenolic antioxidants. J. Org. Chem. 1989, 54, 557-560.

24. Fang, H.Y.; Chiou, S.F.; Uvarani, C.; Wen, Z.H.; Hsu, C.H.; Wu, Y.C.; Wang, W.L.; Liaw, C.C.; Sheu, J.H. Cytotoxic, anti-inflammatory, and antibacterial sulfur-containing polybromoindoles from the Formosan red alga Laurencia brongniartii. Bull. Chem. Soc. Jpn. 2014, 87, 1278-1280.

25. Numata, A.; Kanbara, S.; Takahashi, C.; Fujiki, R.; Yoneda, M.; Usamai, Y.; Fujita, E. A cytotoxic principle of the brown alga Sargassum tortile and structures of chromenes. Phytochemistry 1992, 31, 1209-1213.

26. Iwata, N.; Kitanaka, S. Tetracyclic chromane derivatives from Rhododendron anthopogonoides. J. Nat. Prod. 2010, 73, 1203-1206.

27. Nunez, V.; Castro, V.; Murillo, R.; Ponce-Soto, L.A.; Merfort, I.; Lomonte, B. Inhibitory effects of Piper umbellatum and Piper peltatum extracts towards myotoxic phospholipases A2 from Bothrops snake venoms: Isolation of 4-nerolidylcatechol as active principle. Phytochemistry $\mathbf{2 0 0 5}$, 66, 1017-1025.

28. Alley, M.C.; Scudiero, D.A.; Monks, A.; Hursey, M.L.; Czerwinski, M.J.; Fine, D.L.; Abbott, B.J.; Mayo, J.G.; Shoemaker, R.H.; Boyd, M.R. Feasibility of drug screening with panels of human tumor cell lines using a microculture tetrazolium assay. Cancer Res. 1988, 48, 589-601. 
29. Scudiero, D.A.; Shoemaker, R.H.; Paull, K.D.; Monks, A.; Tierney, S.; Nofziger, T.H.; Currens, M.J.; Seniff, D.; Boyd, M.R. Evaluation of a soluble tetrazolium/formazan assay for cell growth and drug sensitivity in culture using human and other tumor cell lines. Cancer Res. 1988, 48, 4827-4833.

30. Shafi, M.S. Determination of antimicrobial MIC by paper diffusion method. J. Clin. Path. 1975, 28, 989-992.

31. Tello, E.; Castellanos, L.; Arevalo-Ferro, C.; Duque, C. Cembranoid diterpenes from the Caribbean sea whip Eunicea knighti. J. Nat. Prod. 2009, 72, 1595-1602.

32. Yu, H.P.; Hsieh, P.W.; Chang, Y.J.; Chung, P.J.; Kuo, L.M.; Hwang, T.L. 2-(2-Fluorobenzamido) benzoate ethyl ester (EFB-1) inhibits superoxide production by human neutrophils and attenuates hemorrhagic shock-induced organ dysfunction in rats. Free Radic. Biol. Med. 2011, 50, 1737-1748.

33. Hwang, T.L.; Wang, C.C.; Kuo, Y.H.; Huang, H.C.; Wu, Y.C.; Kuo, L.M.; Wu, Y.H. The hederagenin saponin SMG-1 is a natural FMLP receptor inhibitor that suppresses human neutrophil activation. Biochem. Pharmacol. 2010, 80, 1190-1200.

34. Hwang, T.L.; Leu, Y.L.; Kao, S.H.; Tang, M.C.; Chang, H.L. Viscolin, a new chalcone from Viscum coloratum, inhibits human neutrophil superoxide anion and elastase release via a cAMP-dependent pathway. Free Radic. Biol. Med. 2006, 41, 1433-1441.

(C) 2014 by the authors; licensee MDPI, Basel, Switzerland. This article is an open access article distributed under the terms and conditions of the Creative Commons Attribution license (http://creativecommons.org/licenses/by/4.0/). 